\title{
An adaptive algorithm for nonlinear system identification
}

\author{
D C REDDY and K DEERGHA RAO
}

Research and Training Unit for Navigational Electronics, Osmania University, Hyderabad 500007, India

\begin{abstract}
There are several methods - fixed, adaptive, recursive - for the identification of linear and bilinear systems from input-output measurements that are noisy. However, literature is rather scarce as far as such techniques are concerned for the identification of nonlinear systems. The objective of this paper, therefore, is to suggest an iterative technique for the identification of nonlinear system parameters from measurements that are noisy. This technique requires the transformation of a nonlinear system in the state variable form into an input-output autoregressive moving average exogenous (ARMAX) model. The pseudo linear regression algorithm, which has been extensively used for the identification of linear systems, can then be used to identify the nonlinear system parameters. Using this technique simulation studies were carried out which, indeed, confirm the efficacy of the method.
\end{abstract}

Keywords. Nonlinear systems; recursive; identification.

\section{Introduction}

The problem of parameter identification of linear systems from input-output measurements that are noisy has received considerable attention (Astrom \& Eykhoff 1971; Mehra 1971; Iserman \& Bauer 1974). A two-step identification approach using correlation analysis and least squares estimation for these was developed in Mehra (1971) and Iserman \& Bauer (1974). The same methodology was used by Haber (1988) for the identification of nonlinear dynamic systems satisfying the Hammerstein model. Mention has also been made (Billings \& Fakhouri 1978; Haber 1985) of using cross-correlation functions as a simple test of linearity/nonlinearity of a system. Here, it may be mentioned that these methods yield an input-output model and no internal description of the system is available Baheti et al (1980) have extended the same two-step approach for the identification of discrete time bilinear systems by first transforming a canonical state variable model into an equivalent input-output relation and then identifying the unknown parameters, in the presence of noise, by using the first- and second-order statistics.

Such a method could conceivably be extended to the identification of nonlinear systems, given their internal description (state space representation). But this would entail the use of second-order statistics of various correlation functions.

Now, in practical system implementations, second-order statistics can only be 
estimated from the available data, which is often a source of computational delay or error or both. Therefore the need for alternate methods. A new technique that does not suffer from this shortcoming is proposed here. The method envisages the representation of a nonlinear system in the state variable form, which is then cast into an ARMAX input-output model. The system parameters are next estimated from the noisy measurements by the use of a recursive identification algorithm known as the pseudo linear regression (PLR) algorithm (Ljung \& Soderstrom 1986).

\section{A generalized expression for the representation of a nonlinear system in state space}

Since a large body of recursive techniques exists for the identification of linear syctem parameters from noisy data, it would be desirable to see whether these could be extended for the identification of nonlinear systems. The methods invariably assume the linear regression model

where

$$
y(k)=\theta^{T} \phi(k)+v(k)
$$

$\theta^{T}=\left[\theta_{1}, \theta_{2}, \ldots, \theta_{n}\right]$ is known as the parameter vector,

$\phi^{r}(k)=\underset{\text { vector, }}{[y(k-1), y(k-2), \ldots, y(k-n)] \text { is referred to as the regression }}$

$y(k)$ is the output of the system,

and

$v(k)$ is a white noise sequence.

Such models have been widely studied in statistics and the standard least squares method is a natural and efficient way of estimating the parameter vector $\theta$. The estimated parameter vector approaches the true model parameter vector as the number of recursions becomes very large if the noise is white with zero mean. However, if the noise is non-white but small compared to the regression vector, then the estimated parameter vector will be 'close' to the true parameter vector. Considering the usefulness of this method, it is tempting to cast other models, that are not true linear regressions in the form of (1). Thus it would be our endeavour to cast the nonlinear discrete system

where

$$
\begin{aligned}
X(k) & =A X(k-1)+B u(k-1)+\sum_{i=2}^{m} N_{i}[[1,0,0, \ldots 0] X(k-1)]^{i}, \\
y(k) & =C^{T} X(k)
\end{aligned}
$$

$$
\begin{aligned}
A & =\left[\begin{array}{cccccc}
-a_{n-1} & 1 & 0 & \cdots & 0 & 0 \\
-a_{n-2} & 0 & 1 & \cdots & 0 & 0 \\
\vdots & & & & & \\
-a_{1} & 0 & 0 & \cdots & 0 & 1 \\
-a_{0} & 0 & 0 & \cdots & 0 & 0
\end{array}\right], \\
B & =\left[b_{n-1}, b_{n-2}, \ldots, b_{1}, b_{0}\right]^{T}, \\
C & =[1,0,0, \ldots, 0]^{T}, \\
N_{2} & =\left[N_{2, n-1}, N_{2, n-2}, \ldots, N_{2,1}, N_{2,0}\right]^{T},
\end{aligned}
$$




$$
\begin{aligned}
& N_{3}=\left[N_{3, n-1}, N_{3, n-2}, \ldots, N_{3,1}, N_{3,0}\right]^{T}, \\
& \vdots \\
& N_{m}=\left[N_{m, n-1}, N_{m, n-2}, \ldots, N_{m, 1}, N_{m, 0}\right]^{T},
\end{aligned}
$$

into the form of (1)

Observe that (2) represents a very large class of nonlinear systems that are characterized by polynomial nonlinearities which are good approximations of most of the nonlinear phenomena that occur in real life situations, the exceptions being the violent (abrupt) and memory type of nonlinearities

\section{Problem statement}

Consider the system model

where

$$
\begin{aligned}
X(k) & =A X(k-1)+B u(k-1)+\sum_{i=2}^{m} N_{i}[[1,0,0, \ldots, 0] X(k-1)]^{i}, \\
z(k) & =C X(k)+v(k) \quad k=0,1,2, \ldots
\end{aligned}
$$

$X(k)$ is the $n$ dimensional state vector,

$u(k)$ is a stationary stochastic input sequence,

$y(k)$ is a stationary stochastic output sequence,

$A$ is an $n \times n$ matrix,

$B$ is an $n \times 1$ vector,

$C$ is an $1 \times n$ vector,

$N_{i}$ is an $n \times 1$ vector,

$v(k)$ is a white noise sequence with mean and variance given by the equations

$E[v(k)]=0 \quad E[v(k) v(j)]=\gamma \delta_{k j}$,

$\delta_{k j}$ denotes the Kronecker delta function

and $A, B, C$ and $N_{i}$ are the same as given in (2) and $\gamma$ is assumed finite.

It is now required to estimate the unknown but constant parameter matrices $A$, $B, C$ and $N_{i}$ from input-output observations that are noisy.

Towards this end, it is proposed to extend the existing PLR technique of linear system identification to nonlinear systems. However such a procedure would call for casting the state space model of (3) into an input-output ARMAX model of (1).

After a little algebra, it can be shown that (4) can be transformed into the input-output model given by the equation

$$
\begin{gathered}
z(k)=Z^{\mathrm{T}}(k-n) a+U^{\mathrm{T}}(k-n) B+Z^{2^{\mathrm{T}}}(k-n) N_{2}+Z^{3^{\mathrm{T}}}(k-n) N_{3}+\cdots \\
+Z^{m^{\mathrm{T}}}(k-n) N_{m}+v(k),
\end{gathered}
$$


where

$$
\begin{aligned}
Z^{T}(k-n)= & {[(z(k-1)-v(k-1)),(z(k-2)-v(k-2)), \ldots,} \\
& (z(k-n)-v(k-n))], \\
U^{T}(k-n)= & {[u(k-1), u(k-2), \ldots, u(k-n)], } \\
Z^{2^{T}}(k-n)= & {\left[(z(k-1)-v(k-1))^{2},(z(k-2)-v(k-2))^{2}, \ldots,\right.} \\
& \left.(z(k-n)-v(k-n))^{2}\right] \\
Z^{3^{\mathrm{T}}}(k-n)= & {\left[(z(k-1)-v(k-1))^{3},(z(k-2)-v(k-2))^{3}, \ldots,\right.} \\
\left.\quad(z(k-n)-v(k-n))^{3}\right] & \\
\vdots \quad & \left.(z(k-n)-v(k-n))^{m}\right] \\
Z^{m^{T}}(k-n)= & {\left[(z(k-1)-v(k-1))^{m},(z(k-2)-v(k-2))^{m}, \ldots,\right.} \\
a^{T}= & {\left[-a_{n-1},-a_{n-2}, \ldots,-a_{1},-a_{0}\right] }
\end{aligned}
$$

$B, N_{2}, N_{3}$ and $N_{m}$ are the same as given in (2). Note that although (5) represents a nonlinear system yet it is linear in parameters.

\section{The PLR algorithm}

The ARMAX model of (5) can next be rewritten as

where

$$
z(k)=\theta^{r} Z_{1}(k)+v(k)
$$

$$
\begin{aligned}
& Z_{1}(k)=[(z(k-1)-v(k-1)),(z(k-2)-v(k-2)), \ldots,(z(k-n)-v(k-n)), \\
&(z(k-1)-v(k-1))^{2},(z(k-2)-v(k-2))^{2}, \ldots, \\
&(z(k-n)-v(k-n))^{2},(z(k-1)-v(k-1))^{3}, \\
&(z(k-2)-v(k-2))^{3}, \ldots,(z(k-n)-v(k-n))^{3}, \ldots, \\
&(z(k-1)-v(k-1))^{m},(z(k-2)-v(k-2))^{m}, \ldots, \\
&\left.(z(k-n)-v(k-n))^{m}, u(k-1), \ldots, u(k-n)\right]^{T}, \\
& \theta=[- a_{n-1},-a_{n-2}, \ldots,-a_{1},-a_{0}, N_{2, n-1}, N_{2, n-2}, \ldots, \\
& N_{2,0}, N_{3, n-1}, N_{3, n-2}, \ldots, N_{3,0}, \ldots, N_{m, n-1}, N_{m, n-2}, \ldots, \\
&\left.N_{m, 0}, b_{n-1}, b_{n-2}, \ldots, b_{1}, b_{0}\right]^{T} .
\end{aligned}
$$

Note that (6) is formally equivalent to the AR model representation. However, the standard least squares method can not be used for estimating the parameter vector $\theta$ since the regression vector $Z_{1}(k)$ includes unobservable variables $v(k), v(k-1)$, $v(k-n)$ which can only be estimated from the data. The combined procedure of estimating $\theta$ and reconstructing the unobserved $Z_{1}(k)$ components is called the PLR method and was introduced by Solo in 1978 (Ljung \& Soderstrom 1986).

Now let

$$
\hat{v}(k)=z(k)-\hat{\theta}^{T} \hat{Z}_{1}(k)
$$


where

$$
\begin{aligned}
\hat{Z}_{1}(k)= & {[(z(k-1)-\hat{v}(k-1)),(z(k-2)-\hat{v}(k-2)), \ldots,} \\
& (z(k-n)-\hat{v}(k-n)),(z(k-1)-\hat{v}(k-1))^{2},(z(k-2)-\hat{v}(k-2))^{2}, \ldots, \\
& (z(k-n)-\hat{v}(k-n))^{2},(z(k-1)-\hat{v}(k-1))^{3},(z(k-2)-\hat{v}(k-2))^{3}, \ldots, \\
& (z(k-n)-\hat{v}(k-n))^{3}, \ldots,(z(k-1)-\hat{v}(k-1))^{m}, \\
& (z(k-2)-\hat{v}(k-2))^{m}, \ldots,\left(z(k-n)-\hat{v}(k-n)^{m}, u(k-1), \ldots, u(k-n)\right]^{T} .
\end{aligned}
$$

In view of (7), the PLR algorithm (Ljung \& Soderstrom 1986) for the identification of system parameters of the nonlinear system (5), may be written as:

where

$$
\begin{aligned}
\hat{\theta}(k)= & \hat{\theta}(k-1)+L(k)\left[z(k)-\hat{Z}_{1}^{T}(k) \hat{\theta}(k-1)\right], \\
L(k)= & L(k-1) \hat{Z}_{1}(k)\left[\lambda(k)+\hat{Z}_{1}^{T}(k) P(k-1) \hat{Z}_{1}(k)\right]^{-1}, \\
P(k)= & P(k-1)-P(k-1) \hat{Z}_{1}(k)\left[\lambda(k)+\hat{Z}_{1}^{T}(k) P(k-1) \hat{Z}_{1}(k)\right]^{-1} \times \\
& \quad \times \hat{Z}_{1}^{T}(k) P(k-1),
\end{aligned}
$$

$L(k)$ is the Kalman gain vector,

$P(k)$ is the covariance matrix,

$$
\lambda(k)=\lambda_{0} \lambda(k-1)+0 \cdot 01
$$

with

$$
\lambda_{0}=0.99 \text { and } \lambda(0)=0.95 \text {. }
$$

The iterative algorithm may be initialized by setting

and

$$
\hat{\theta}(0)=0
$$

$$
P(0)=\alpha I
$$

where $\alpha$ is some sufficiently large positive number. For convergence of the algorithm see Ljung \& Soderstrom (1986).

\section{Simulation studies}

Using the proposed method of identification and the PLR algorithm, simulation studies were carried out for the identification of two nonlinear discrete systems that are given below.

Example 1: Assume a state variable model with measurement noise $v(k)$.

and

$$
\begin{gathered}
X(k)=\left[\begin{array}{cc}
-1.5 & 1 \\
-0.56 & 0
\end{array}\right] X(k-1)+\left[\begin{array}{l}
1.0 \\
0.5
\end{array}\right] u(k-1)+ \\
+\left[\begin{array}{r}
-0.6 \\
-0.5
\end{array}\right][[1,0] X(k-1)]^{2}
\end{gathered}
$$

$$
z(k)=\left[\begin{array}{ll}
1 & 0
\end{array}\right] X(k)+v(k)
$$


An ARMAX model corresponding to (9) is given by the equation

$$
\begin{aligned}
z(k)=- & 1.5(z(k-1)-v(k-1))-0.56(z(k-2)-v(k-2))- \\
& -0 \cdot 0(z(k-1)-v(k-1))^{2}-0.5(z(k-2)-v(k-2))^{2}+ \\
& +u(k-1)+0.5 u(k-2)+v(k) .
\end{aligned}
$$

The input $\{u(k)\}$ was assumed to be a pseudo random binary sequence while the noise sequence $\{v(k)\}$ was white with zero mean. The parameter estimates were obtained by applying the PLR algorithm to a 300-point data sequence with $S / N=60 \mathrm{~dB}$. These are as shown in figure 1 . From these it may be observed that the parameter estimates are in close agreement with the assumed values.

Example 2: Consider next a state variable model with measurement noise $v(k)$,

$$
\begin{aligned}
X(k)= & {\left[\begin{array}{lrr}
-2.0 & 1.0 & 0 \\
-1.31 & 0 & 1.0 \\
-0.28 & 0 & 0
\end{array}\right] X(k-1)+\left[\begin{array}{l}
1.0 \\
0.5 \\
0.0
\end{array}\right] u(k-1)+} \\
& +\left[\begin{array}{l}
-0.6 \\
-0.5 \\
-0.15
\end{array}\right][[1,0,0] X(k-1)]^{2}
\end{aligned}
$$

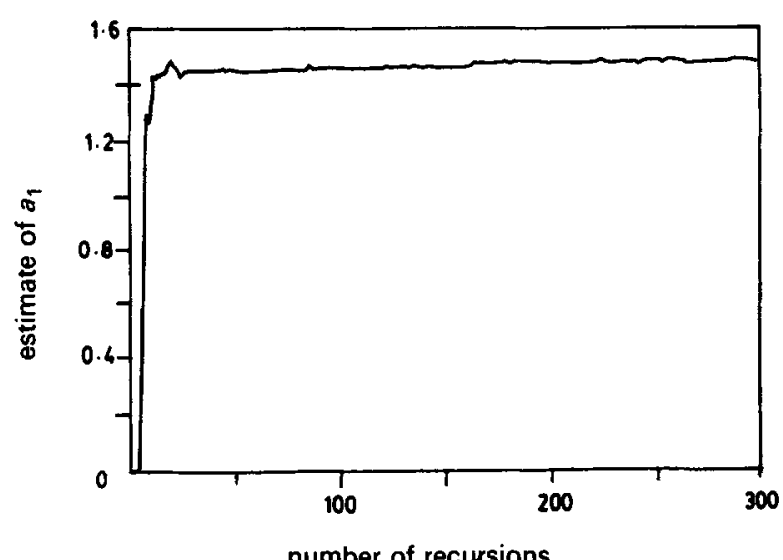

number of recursions

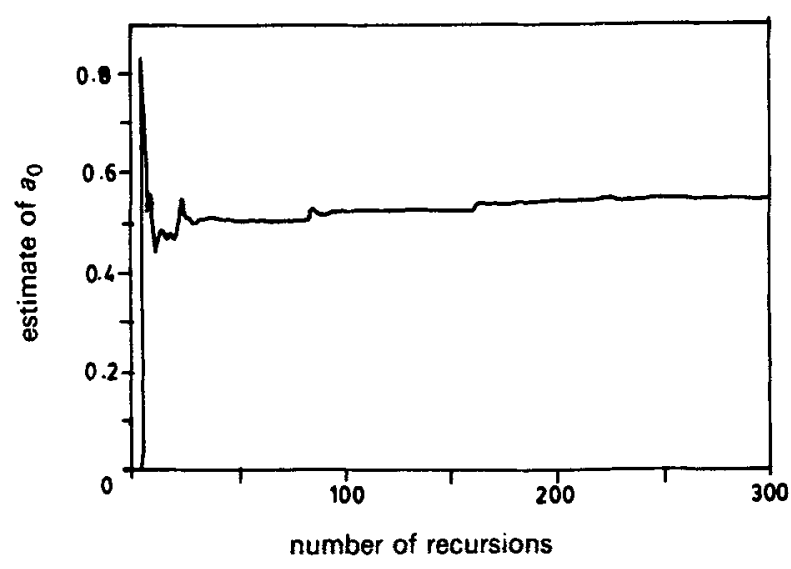

Figure 1.(a) Recursions versus estimate of parameter $a_{1}$.

Figure 1.(b) Recursions versus estimate of parameter $a_{0}$. 

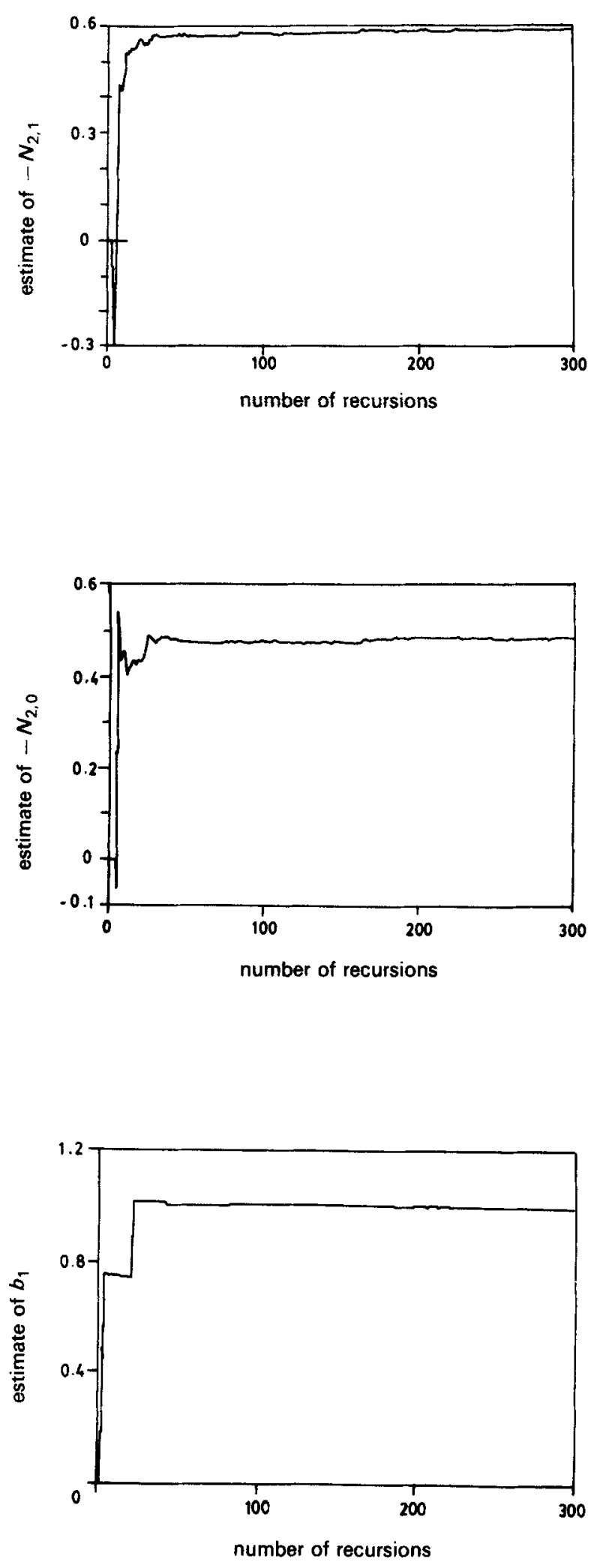

Figure 1.(c) Recursions versus estimate of parameter $-N_{2,1}$.

Figure 1.(d) Recursions versus estimate of parameter $-N_{2,0}$.
Figure 1.(e) Recursions versus estimate of parameter $b_{1}$. 


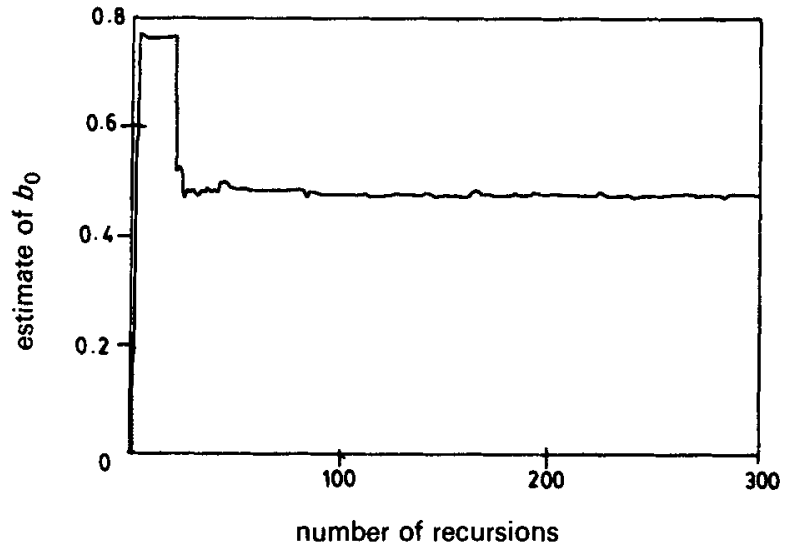

Figure 1.(f) Recursions versus estimate of parameter $b_{0}$.

and

$$
z(k)=[1,0,0] X(k)+v(k) .
$$

Equation (11) can be transformed into an input-output ARMAX model given by the equation:

$$
\begin{aligned}
& z(k)=-2 \cdot 0(z(k-1)-v(k-1))-1 \cdot 31(z(k-2)-v(k-2))- \\
&-0 \cdot 28(z(k-3)-v(k-3))+u(k-1)+0 \cdot 5 u(k-2)- \\
&-0 \cdot 6(z(k-1)-v(k-1))^{2}-0 \cdot 5(z(k-2)-v(k-2))^{2}- \\
&-0 \cdot 15(z(k-3)-v(k-3))^{2}+v(k),
\end{aligned}
$$

which can then be cast into the form of (6) to which the PLR algorithm is directly applicable. The input sequence $\{u(k)\}$ and the noise sequence $\{v(k)\}$ are the same as in the first example. The parameter estimates were then obtained by using the PLR algorithm to a 300 -point data sequence with $S / N=60 \mathrm{~dB}$. The simulation results are as shown in figure 2 . Observe the close agreement between the estimated and assumed values of the parameters.

From figures 1 and 2 it is observed that the error is large initially as expected since all the parameters are assumed to be zero. However, as the number of recursions increase the error decreases rapidly and the estimated parameters approach true

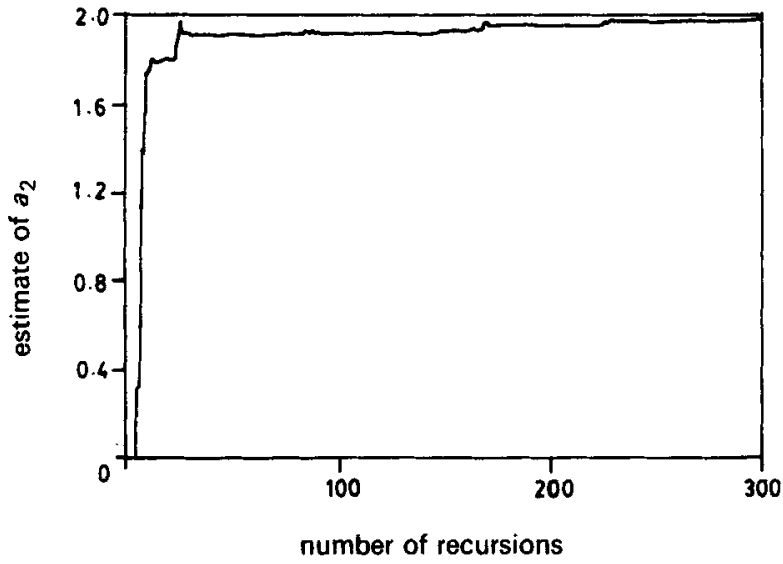

Figure 2.(a) Recursions versus estimate of parameter $a_{2}$. 

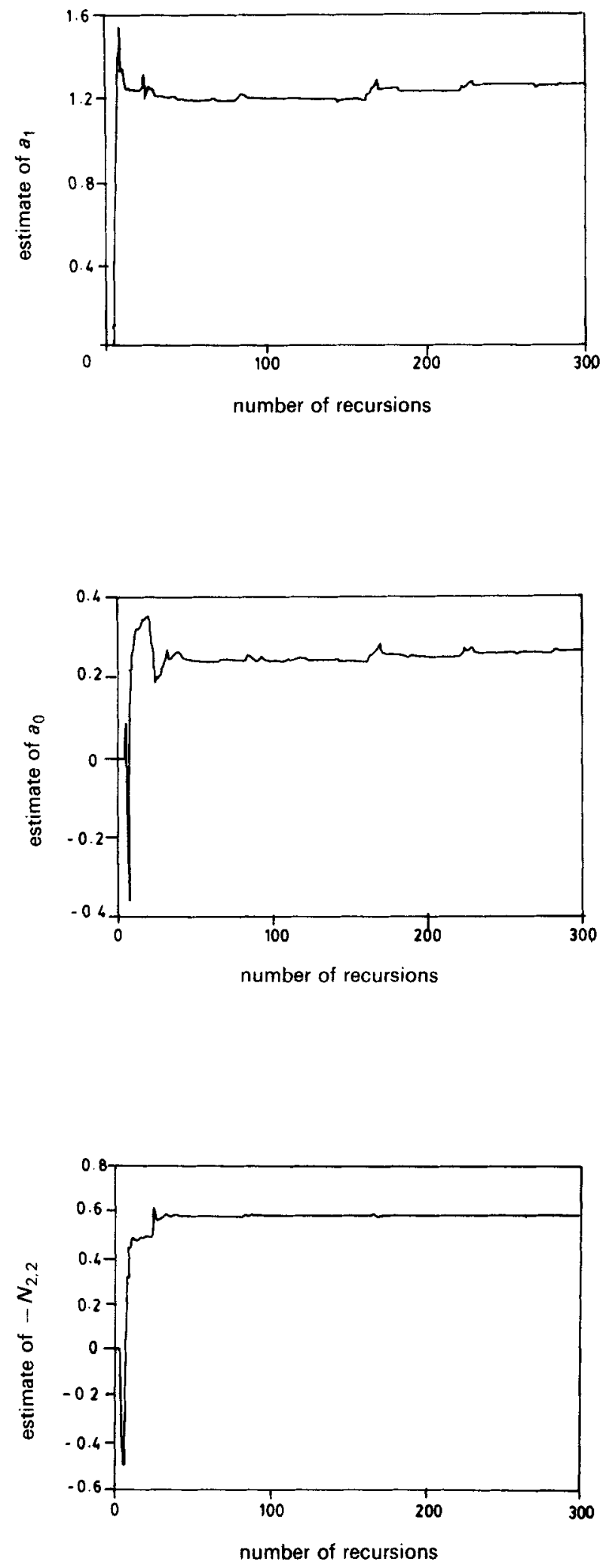

Figure 2.(b) Recursions versus estimate of parameter $a_{1}$.

Figure 2.(c) Recursions versus estimate of parameter $a_{0}$.
Figure 2.(d) Recursions versus estimate of parameter $-N_{2,2}$. 

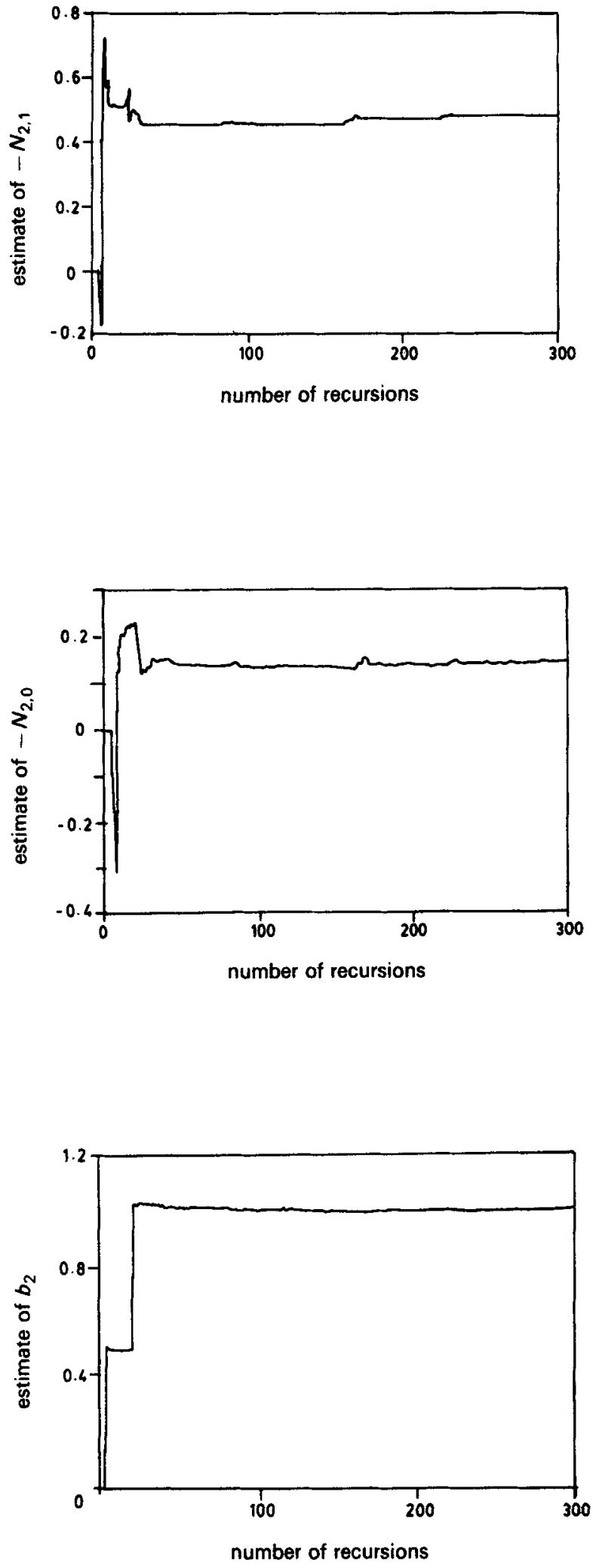

Figure 2.(e) Recursions versus estimate of parameter $-N_{2.1}$

Figure 2.(f) Recursions versus estimate of parameter $-N_{2,0}$.

Figure 2.(g) Recursions versus estimate of parameter $b_{2}$. 

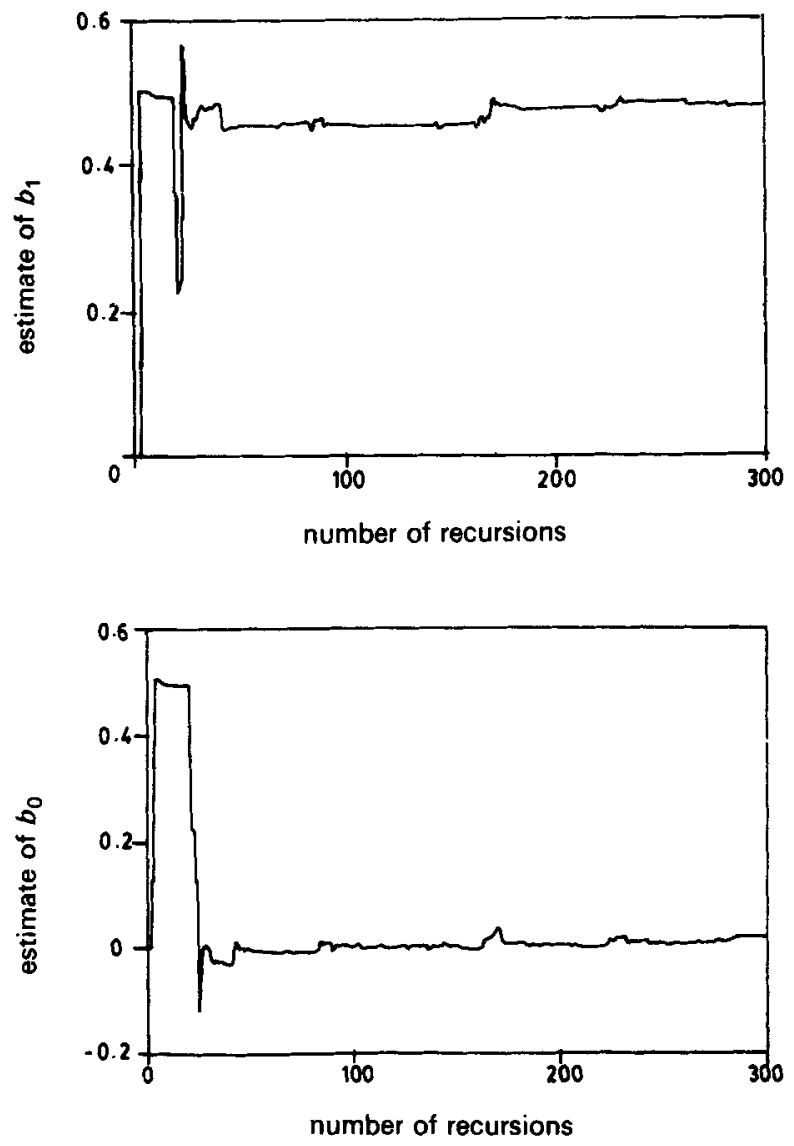

Figure 2.(h) Recursions versus estimate of parameter $b_{1}$.

Figure 2(i) Recursions versus estimate of parameter $b_{0}$.

parameters. But this is not a smooth process as is evident from the kinks in the graphs. The fact that the least square error rather than the mean square error is being minimised, accounts for the rapid variations.

\section{Conclusions}

In this paper a generalized expression for the state space representation of a nonlinear system has been developed. This enables the extension of the PLR algorithm, used extensively for the identification of linear systems, to that of nonlinear system parameters. But it requires that the system be first transformed into an input-output ARMAX model. The efficacy of using the pseudo linear regression algorithm for the identification of parameters of a nonlinear system has been demonstrated through simulation studies. These studies show that the parameters converge to the true values when the $S / N$ is 'high'.

\section{References}

Astrom K J, Eykhoff P 1971 System identification - A survey. Automatica 7: 123-162

Baheti R S, Mohler R R, Spang H A III 1980 Second-order correlation method for bilinear system identification. IEEE Trans. Autom. Control AC-25: 1141-1146 
Billings S A, Fakhouri S Y 1978 Theory of separate processes with application to the identification of nonlinear systems. Proc. Inst. Electr. Eng. 129: 1051-1058

Haber R 1985 Nonlinearity tests for dynamic processes. 7th IF AC Symposium on identification and system parameter estimation, York, UK, pp. 409-413

Haber R 1988 Parametric identification of nonlinear dynamic systems based on nonlinear cross correlation functions. Proc. Inst. Electr. Eng. 135: 405-420

Isermann R, Bauer U 1974 Two-step process identification with correlation analysis and least squares parameter estimation. Trans. ASME, J. Dynamic System, Measure Control pp. 426-432

Ljung L, Soderstrom T 1986 Theory and practice of recursive identification (Boston: MIT)

Mehra R K 1971 On-line identification of linear dynamic systems with application to Kalman filtering. IEEE Trans. Autom. Control AC-16: 12-21 